\title{
DESENVOLVIMENTO DO MÉTODO DE DATAÇÃO QUÍMICA U-Th-Pb DE MONAZITA POR MICROSSONDA ELETRÔNICA NA UFMG
}

\author{
Alexandre de Oliveira Chaves ${ }^{1}$, Elizabeth Kerpe de Oliveira ${ }^{2}$, Luiz Rodrigues Armoa Garcia ${ }^{3}$ \\ ${ }^{1}$ Centro de Pesquisas Manoel Teixeira da Costa - Departamento de Geologia - Instituto de Geociências - UFMG - BH - MG - \\ alochaves@yahoo.com.br \\ ${ }^{2}$ Centro de Desenvolvimento da Tecnologia Nuclear - CDTN/CNEN - BH - MG \\ ${ }^{3}$ Laboratório de de Microanálises - Departamento de Física - UFMG - BH - MG
}

Recebido em 7 de agosto de 2013; aceito em 27 de dezembro de 2013

\begin{abstract}
RESUMO: O método de datação química U-Th-Pb (não-isotópica) de monazita por microssonda eletrônica vem sendo desenvolvido há pelo menos 20 anos e já tem o reconhecimento da comunidade geológica por apresentar resultados que se equivalem à geocronologia isotópica $\mathrm{U}-\mathrm{Pb}$. Este mineral contém quantidades negligenciáveis de chumbo comum, guardando apenas $\mathrm{Pb}$ radiogênico proveniente do Th e $\mathrm{U}$ deste mineral. O desenvolvimento deste método no Laboratório de Microanálises do Departamento de Física da Universidade Federal de Minas Gerais mostra que os dados químicos de $\mathrm{U}$, Th e $\mathrm{Pb}$ de cristais de monazita fornecidos por sua microssonda eletrônica produzem idades não-isotópicas para eles que se equiparam às idades isotópicas U-Pb produzidas pela técnica LA-ICP-MS. Grãos de monazita de placers marinhos de Buena (RJ) isotopicamente datados pelo método U-Pb com idades entre 530 e 580 Ma foram quimicamente datadas na UFMG entre 505 e 580 Ma. Estes resultados são consideravelmente compatíveis e colocam o referido laboratório a disposição da comunidade geocientífica para obtenção de idades de cristais de monazita.
\end{abstract}

\section{Palavras-Chave: MONAZITA, DATAÇÃO QUÍMICA, MICROSSONDA ELETRÔNICA, UFMG}

ABSTRACT: DEVELOPMENT OF THE MONAZITE U-Th-Pb CHEMICAL DATING METHOD BY USING ELECTRON MICROPROBE AT UFMG. The monazite U-Th-Pb chemical dating method (non-isotopic) by electron microprobe has been developed for about 20 years and has the acceptance of the geological community by presenting results that are equivalent to the isotope $\mathrm{U}$-Pb geochronology. This mineral contains negligible amounts of common lead, keeping only radiogenic $\mathrm{Pb}$ from the Th and $\mathrm{U}$ of this mineral. The development of this method in the microanalysis laboratory of the Physics Department- UFMG shows that the monazite $U$, Th and Pb chemical data provided by its microprobe produce non-isotopic ages for it that are similar to the U-Pb isotopic ages produced by LA-ICP-MS technique. Monazite grains from marine placers of Buena (RJ) isotopically dated by method U-Pb between 530 and 580 Ma were chemically dated at UFMG between 505 and $580 \mathrm{Ma}$. These results are consistent each other and put the laboratory available to the geoscience community as a tool in obtaining monazite ages.

Keywords: MONAZITE, CHEMICAL DATING, ELECTRON MICROPROBE, UFMG

\section{INTRODUÇÃO}

Monazita, um fosfato de elementos terras-raras leves, é um mineral acessório difundido em diversas litologias de composição meta a peraluminosa, incluindo granitóides e pegmatitos, além de xistos e gnaisses paraderivados que variam desde o facies xisto-verde a granulito. Concentra-se também em depósitos sedimentares do tipo placer em associação a outros minerais pesados (Overstreet, 1967). A monazita é portadora de $T h, U$ e do $\mathrm{Pb}$ essencialmente radiogênico derivado de ambos por decaimento radioativo. Há incorporação negligenciável de $\mathrm{Pb}$ comum no crescimento natural de seus cristais (Parrish, 1990), eliminando-se a necessidade de correção isotópica robusta para o mesmo em estudos geocronológicos. Desde os anos 90 , este mineral tem se tornado reconhecidamente importante na definição não só da idade de cristalização magmática, como também de eventos de metamorfismo e deformação, idades estas obtidas através de análises não-destrutivas, precisas e de alta resolução espacial de $\mathrm{U}$, Th e $\mathrm{Pb}$ por microssonda eletrônica. Uma vez que cristais de monazita podem ser internamente homogêneos ou heterogêneos em função de sua história geológica, as imagens de elétrons retro-espalhados fornecidas pela microssonda são capazes de revelar eventuais domínios/zoneamentos composicionais em cristais de monazita, os quais podem ser cronologicamente ordenados pelo método de datação química para produzir informações termotectônicas sobre o crescimento de cristais e padrões de recristalização relacionados a reações metamórficas ou microestruturas (Suzuki e Adachi, 1991; Montel et al., 1996; Williams et al., 1999; Foster et al., 2004; Dahl et al., 2005; Pyle et al., 2005;. Williams et al., 2007; Vlach, 2010). As análises em microssonda eletrônica são facilmente obtidas a partir da mesma lâmina delgada ou seção polida utilizadas nos estudos petrográficos, desde que sejam extremamente bem polidas para uma perfeita excitação sobre uma superfície plana.

Tendo em vista que a Universidade Federal de Minas Gerais (UFMG) não dispõe de laboratórios de geocronologia isotópica, o Laboratório de Microanálises (LMA) do Departamento de Física desta universidade, por meio de sua microssonda eletrônica, passa a ser uma alternativa para estudos geocronológicos não isotópicos de monazita. O objetivo deste artigo é apresentar uma comparação direta entre idades isotópicas obtidas no Laboratório de Geocronologia Isotópica da Universidade de Brasília através da técnica LA-ICP-MS (Laser Ablation Inductively Coupled Plasma Mass Spectrometry) e idades químicas cuidadosamente obtidas no LMA para cristais de monazita, no intuito de apresentar a confiabilidade das suas idades não isotópicas obtidas a partir do desenvolvimento da técnica de sua 
datação química por microssonda eletrônica na UFMG.

\section{AMOSTRAS E MÉTODOS}

Os grãos de monazita investigados nesta pesquisa foram coletados em um concentrado final constituído pelo produto da lavra de várias jazidas de placers marinhos explotadas pelas Indústrias Nucleares do Brasil (INB) na região de Buena, litoral norte fluminense, município de São Francisco de Itabapoana, RJ. Uma amostra do concentrado puro de monazita (grãos desagregados) com 20 quilos foi reduzida, em sucessivas etapas de homogeneização e quarteamento, visando separar alíquotas representativas com cerca de 3 gramas, nas quais grãos individualizados selecionados foram montados em lâminas delgadas e blocos de resina, submetidos ao desbaste e polimento até se obter uma superfície com qualidade adequada para petrografia, determinações isotópicas por LA-ICP-MS e análises quantitativas na microssonda eletrônica.

O estudo das características microscópicas da monazita, em lupa e microscópio óptico petrográfico polarizador, com luz transmitida e refletida, que levou à seleção dos grãos aqui utilizados, foi realizado no Laboratório de Inclusões Fluídas e Metalogênese, do Centro de Desenvolvimento da Tecnologia Nuclear (CDTN/CNEN). Grãos de monazita internamente homogêneos, livres de domínios composicionais distintos, foram separados dos heterogêneos e escolhidos para as investigações geocronológicas tanto isotópicas quanto químicas.

Análises isotópicas in situ de U-Pb dos grãos de monazita homogêneos por LA-ICP-MS, segundo o procedimento analítico descrito em Bühn et al. (2009), foram realizadas no Laboratório de

Tabela 1- Parâmetros para análises pontuais quantitativas WDS utilizados pela microssonda eletrônica do LMA-UFMG.

\begin{tabular}{|c|c|c|c|c|c|c|c|c|}
\hline \multicolumn{9}{|c|}{ Método $=$ WDS } \\
\hline \multicolumn{9}{|c|}{ Intensidade da corrente $=50 \mathrm{nA}$} \\
\hline \multicolumn{9}{|c|}{ Diâmetro do feixe de elétrons $=2$ micrômetros } \\
\hline \multicolumn{9}{|c|}{ Tempo de contagem offsets background } \\
\hline Elemento & Raio-X & Cristal & Pico & Backg & round & $\mathrm{Bg} \mathrm{L}$ & $\mathrm{Bg} \mathrm{O}$ & Padrão analítico \\
\hline $\mathrm{Y}$ & $\mathrm{La}$ & TAP & 20,0 & 10,0 & (seg) & 1,12 & 0,84 & YAG - Granada de $Y$ e $A l$ \\
\hline Dy & $\mathrm{Lb}$ & LIF & 20,0 & 10,0 & $(\mathrm{seg})$ & 0,84 & 0,00 & $\mathrm{DyPO}_{4}$ \\
\hline $\mathrm{P}$ & $\mathrm{Ka}$ & PETJ & 10,0 & 5,0 & $(\operatorname{seg})$ & 0,70 & 0,70 & Monazita \\
\hline $\mathrm{Si}$ & $\mathrm{Ka}$ & TAP & 10,0 & 5,0 & $(\mathrm{seg})$ & 1,38 & 0,84 & $\mathrm{ThSiO}_{4}$ thorita sintética \\
\hline Gd & $\mathrm{Lb}$ & LIF & 20,0 & 10,0 & $(\mathrm{seg})$ & 0,84 & 0,98 & $\mathrm{GdPO}_{4}$ \\
\hline $\mathrm{Pb}$ & $\mathrm{Mb}$ & PETJ & 200,0 & 100,0 & (seg) & 1,64 & 0,64 & Crocoita \\
\hline $\mathrm{Fe}$ & $\mathrm{Ka}$ & LIF & 10,0 & 5,0 & $(\mathrm{seg})$ & 0,70 & 0,84 & Hematita \\
\hline Th & $\mathrm{Ma}$ & PETJ & 20,0 & 10,0 & (seg) & 1,95 & 0,84 & $\mathrm{ThSiO}_{4}$ thorita sintética \\
\hline $\mathrm{Sm}$ & $\mathrm{Lb}$ & LIF & 20,0 & 10,0 & $(\mathrm{seg})$ & 0,55 & 0,53 & REE2 \\
\hline $\mathrm{U}$ & $\mathrm{Mb}$ & PETJ & 150,0 & 75,0 & $(\operatorname{seg})$ & 0,00 & 2,00 & $\mathrm{UO}_{2}$ sintético \\
\hline Mn & $\mathrm{Ka}$ & LIF & 20,0 & 10,0 & (seg) & 0,56 & 0,00 & Rodonita \\
\hline $\mathrm{Ca}$ & Ka & PETJ & 10,0 & 5,0 & $(\operatorname{seg})$ & 0,84 & 0,70 & $\mathrm{Ca}_{2} \mathrm{P}_{2} \mathrm{O}_{7}$ apatita sintética \\
\hline $\mathrm{Nd}$ & $\mathrm{Lb}$ & LIF & 20,0 & 10,0 & (seg) & 0,84 & 0,84 & $\mathrm{NdPO}_{4}$ \\
\hline $\mathrm{La}$ & $\mathrm{La}$ & PETJ & 10,0 & 5,0 & $(\mathrm{seg})$ & 0,84 & 0,84 & Monazita \\
\hline $\mathrm{Pr}$ & $\mathrm{Lb}$ & LIF & 20,0 & 10,0 & $(\mathrm{seg})$ & 0,84 & 0,66 & $\mathrm{PrPO}_{4}$ \\
\hline $\mathrm{Ce}$ & $\mathrm{La}$ & LIF & 10,0 & 5,0 & (seq) & 0,84 & 1,12 & Monazita \\
\hline
\end{tabular}

Geocronologia Isotópica da Universidade de Brasília, utilizando um equipamento Thermo-Finnigan Neptune - Multi-collector inductively coupled plasma mass spectrometer (MC-ICP-MS) acoplado com um sistema laser NewWave de $213 \mathrm{~nm}$. O padrão GJ-J de Jackson et al. (2004) foi usado como padrão de referência primária no esquema analítico standardsample bracketing. Para cada grão de monazita foram obtidas quatro análises pontuais com as seguintes feições: diâmetro do feixe de laser (micrômetro): 40; energia do laser (\%): 70 em 3,06 $\mathrm{J} / \mathrm{cm}^{2}$; frequência do laser $(\mathrm{Hz}): 8$; fluxo de $\mathrm{Ar}$ (L/min): 0,84; fluxo de $\mathrm{He}(\mathrm{L} / \mathrm{min}): 0,40$. A redução dos dados incluiu a consideração dos brancos, o cálculo dos erros 2 sigma do desvio padrão, e os valores theta $(\theta)$ conforme Albarède et al. (2004). Mesmo negligenciável em cristais de monazita, por segurança efetuou-se a correção para $\mathrm{Pb}$ comum utilizando os valores de Stacey e Kramers (1975). Diagramas de concórdia U-Pb foram construídos com auxílio do software Isoplot (Ludwig, 2003).

As composições químicas dos grãos de monazita homogêneos abladados a laser foram obtidas no LMA-UFMG, em uma microssonda JEOL, modelo JXA-8900, através de espectrometria por dispersão de comprimento de onda (WDS). Os parâmetros para análises pontuais quantitativas WDS utilizados encontram-se na tabela 1 . Os padrões analíticos empregados foram Monazita, Rodonita, Hematita e Crocoita da Coleção Astimex, YAG, Apatita e Thorita da Coleção lan Steele e os ortofostatos de terras raras do Smithsonian Institute. O modelo utilizado para corrigir os efeitos de matriz em função das diferenças nas composições das amostras e do padrão, descrito em Toya et al. (1984), leva em conta os fatores ZAF. 
Sobreposições de picos de raios- $X$ entre $\mathrm{Y}$ e $\mathrm{Pb}$ não precisaram ser corrigidas por não ter havido medição em PbMa (Chumbo $\mathrm{M}$ alfa), mas apenas em $\mathrm{PbMb}$ (chumbo $\mathrm{M}$ beta). Entretanto, para se evitar erros nas idades obtidas, a interferência de ThMz (tório $\mathrm{M}$ gama) sobre o UMb (urânio $\mathrm{M}$ beta) medido precisou ser corrigida, seguindo Scherrer et al. (2000) em adaptação às condições do LMAUFMG, da seguinte maneira:

\section{$U$ corrigido $=U$ medido $-(0.006365 x$ Th medido $)$.}

O cálculo das idades químicas U-Th- $\mathrm{Pb}$ e dos erros associados foi realizado com o auxílio do software EPMA Dating de Pommier et al. (2004), em cujo manual a sistemática encontra-se explicada. 0 cálculo das médias das idades e de seus parâmetros estatísticos foi obtido com o apoio do software Isoplot (Ludwig, 2003).

\section{RESULTADOS}

Os dados $\mathrm{U}-\mathrm{Pb}$ isotópicos obtidos para 5 grãos de monazita investigados encontram-se na tabela 2 e com eles foram construídos os diagramas de concórdia para os 5 grãos, que estão apresentados na figura 1 juntamente com as suas respectivas imagens de elétrons retro-espalhados, obtidas na microssonda. Estas imagens confirmam a homogeneidade interna dos grãos não zonados investigados.

$\mathrm{Na}$ tabela 3 estão apresentados os resultados das análises químicas dos grãos de monazita investigados, do tipo monazita-(Ce), bem como as suas respectivas idades $\mathrm{U}-\mathrm{Th}-\mathrm{Pb}$ não-isotópicas. A média das idades de cada grão encontra-se nos respectivos diagramas da figura 2 .

Tabela 2-Resultados U-Pb obtidos por Laser Ablation MC-ICP-MS em grãos homogêneos de monazita oriundos de placers marinhos de Buena-RJ.

\begin{tabular}{|c|c|c|c|c|c|c|c|c|c|c|c|c|}
\hline Monaz & $\begin{array}{c}\text { Razão } \\
{ }^{206} \mathrm{~Pb} /{ }^{238} \mathrm{U}^{*}\end{array}$ & $\begin{array}{l}2 \sigma \\
(\%)\end{array}$ & $\begin{array}{c}\text { Razão } \\
{ }^{207} \mathrm{~Pb} /{ }^{235} \mathrm{U}^{*}\end{array}$ & $\begin{array}{l}2 \sigma \\
(\%)\end{array}$ & $\begin{array}{c}\text { Razão } \\
{ }^{207} \mathrm{~Pb} /{ }^{206} \mathrm{~Pb}^{*}\end{array}$ & $\begin{array}{l}2 \sigma \\
(\%)\end{array}$ & $\begin{array}{c}\text { Idade } \\
{ }^{206} \mathrm{~Pb}^{238} \mathrm{U}\end{array}$ & $\begin{array}{c}2 \sigma \\
\text { (Ma) }\end{array}$ & $\begin{array}{c}\text { Idade } \\
{ }^{207} \mathrm{~Pb} /\left.\right|^{235} \mathrm{U}\end{array}$ & $\begin{array}{c}2 \sigma \\
\text { (Ma) }\end{array}$ & $\begin{array}{c}\text { Idade } \\
{ }^{207} \mathrm{~Pb} /{ }^{206} \mathrm{~Pb}\end{array}$ & $\begin{array}{c}2 \sigma \\
(\mathrm{Ma})\end{array}$ \\
\hline 2G1-1 & 0.09151 & 1.56 & 0.76033 & 2.58 & 0.05889 & 1.68 & 563.1 & 14 & 557.5 & 20.4 & 563.1 & 9.5 \\
\hline G1-2 & 0.09467 & 1.37 & 0.76732 & 2.58 & 0.05941 & 1.82 & 581.8 & 12.8 & 563.1 & 20.2 & 582.2 & 10.6 \\
\hline G1-3 & 0.09422 & 1.35 & 0.75914 & 2.46 & 0.05935 & 1.73 & 579.4 & 12.5 & 560.3 & 19.7 & 579.9 & 10 \\
\hline 2G1-4 & 0.09494 & 1.39 & 0.77485 & 2.59 & 0.05945 & 8.24 & 583.2 & 12.9 & 565.3 & 50.2 & 583.5 & 48.1 \\
\hline G2-1 & 0.08622 & 3.43 & 7057 & 5.11 & 05804 & 4.84 & 32.4 & 29.2 & 532.4 & 44.5 & 531.1 & 25.7 \\
\hline G2-2 & 0.0866 & 3.7 & 0.70577 & 6.89 & 0.05811 & 5.16 & 535.1 & 31.7 & 538.9 & 51.4 & 534 & 27.5 \\
\hline $4 \mathrm{G} 2-3$ & 0.0869 & 3.89 & 0.68197 & 8.01 & 0.05821 & 10.64 & 537.0 & 33.4 & 526.3 & 71.1 & 537.5 & 57.2 \\
\hline G1-1 & 0.08837 & 2.68 & 0.68307 & 3.8 & 0.05839 & 1.77 & 545.2 & 23.4 & 520.2 & 30 & 544.4 & 9.6 \\
\hline 6G1-2 & 0.08844 & 2.81 & 0.70284 & 4.06 & 0.0584 & 4.27 & 545.6 & 24.5 & 532 & 37.1 & 544.8 & 23.3 \\
\hline 5G1-3 & 0.08774 & 2.74 & 0.70145 & 4.26 & 0.05829 & 3.86 & 541.5 & 23.7 & 531 & 35.7 & 540.7 & 20.9 \\
\hline 5G1-4 & 0.09161 & 2.65 & 0.72262 & 4.74 & 0.05888 & 8.12 & 564.1 & 23.9 & 540.7 & 52.2 & 562.6 & 45.7 \\
\hline 7G1-1 & 0.08433 & 3.99 & 0.67732 & 4.1 & 0.05769 & 2.11 & 518.6 & 33.6 & 483.3 & 32.4 & 518.1 & 10.9 \\
\hline 7G1-2 & 0.08398 & 3.54 & 0.67793 & 3.71 & 0.05763 & 2.24 & 516.7 & 30.8 & 486.2 & 30.4 & 515.7 & 11.6 \\
\hline 7G1-3 & 0.08538 & 3.26 & 0.68435 & 3.39 & 0.05786 & 1.94 & 524.9 & 28.8 & 488.7 & 27.8 & 524.4 & 10.2 \\
\hline 7G1-4 & 0.08768 & 3.26 & 0.70312 & 3.51 & 0.05822 & 2.22 & 539.0 & 30.4 & 505.8 & 30 & 538.2 & 11.9 \\
\hline $8 \mathrm{G} 2-1$ & 0.08741 & 6.59 & 0.7082 & 9.28 & 0.05832 & 6.25 & 540.0 & 56.9 & 540.5 & 87.2 & 541.7 & 33.9 \\
\hline $8 G 2-2$ & 0.09158 & 4.78 & 0.73503 & 5.81 & 0.05894 & 1.94 & 564.3 & 43.2 & 553 & 50.9 & 564.8 & 10.9 \\
\hline $8 G 2-3$ & 0.08991 & 4.74 & 0.73714 & 5.64 & 0.05866 & 1.84 & 554.3 & 42 & 552.2 & 54.1 & 554.3 & 10.2 \\
\hline $8 G 2-4$ & 0.08807 & 4.48 & 0.69817 & 5.65 & 0.05833 & 1.81 & 543.4 & 38.9 & 528.8 & 46.3 & 542.3 & 9.8 \\
\hline
\end{tabular}

$\left({ }^{*}\right)$ valores corrigidos para ${ }^{204} \mathrm{~Pb}$ comum.

Tabela 3 - Análises químicas dos grãos de monazita investigados e suas respectivas idades químicas (não-isotópicas).

\section{Grão B2G1}

\begin{tabular}{|c|c|c|c|c|c|c|c|c|c|c|c|c|c|c|c|c|c|}
\hline Monaz & $\mathrm{Y}_{2} \mathrm{O}_{3}$ & $\mathrm{Dy}_{2} \mathrm{O}_{3}$ & $\mathrm{P}_{2} \mathrm{O}_{5}$ & $\mathrm{SiO}_{2}$ & $\mathrm{Gd}_{2} \mathrm{O}_{3}$ & $\mathrm{PbO}$ & $\mathrm{FeO}$ & $\mathrm{ThO}_{2}$ & $\mathrm{Sm}_{2} \mathrm{O}_{3}$ & $\mathrm{UO}_{2}$ & $\mathrm{MnO}$ & $\mathrm{CaO}$ & $\mathrm{Nd}_{2} \mathrm{O}_{3}$ & $\mathrm{La}_{2} \mathrm{O}_{3}$ & $\mathrm{Pr}_{2} \mathrm{O}_{3}$ & $\mathrm{Ce}_{2} \mathrm{O}_{3}$ & al \\
\hline 2G1-1 & 0.60 & 0.18 & 24.38 & 3.13 & 0.41 & 0.35 & 0.04 & 13.57 & 1.13 & 0.29 & 0.04 & 0.61 & 8.69 & 14.10 & 2.69 & 29.96 & \\
\hline $201-2$ & 0.50 & 0.12 & 25.62 & 2.71 & 0.42 & 0.32 & 0.02 & 12.30 & 1.01 & 0.28 & 0.01 & 0.62 & 8.70 & 14.44 & 2.79 & 30.09 & \\
\hline $2 \mathrm{G} 1-3$ & 0.51 & 0.14 & 25.47 & 2.89 & 0.38 & 0.34 & 0.00 & 13.26 & 0.94 & 0.29 & 0.01 & 0.60 & 8.57 & 14.29 & 2.69 & 30.43 & 00.8 \\
\hline 2G1-4 & 0.49 & 0.08 & 25.17 & 2.68 & 0.34 & 0.32 & 0.01 & 12.21 & 1.01 & 0.29 & 0.00 & 0.57 & 8.70 & 14.44 & 2.46 & 30.36 & \\
\hline $2 \mathrm{G} 1-5$ & 0.48 & 0.14 & 24.96 & 2.79 & 0.41 & 0.32 & 0.02 & 12.60 & 0.86 & 0.27 & 0.03 & 0.61 & 8.59 & 14.08 & 2.56 & 30.10 & \\
\hline
\end{tabular}

$\begin{array}{lccccccccc}\text { Monaz } & \begin{array}{c}\text { Idade } \\ \text { Ma }\end{array} & \begin{array}{c}2 \sigma \\ \text { Ma }\end{array} & \begin{array}{c}\mathrm{U} p m \\ \text { ppm }\end{array} & \begin{array}{c}2 \sigma \\ \mathrm{ppm}\end{array} & \begin{array}{c}\text { Th } \\ \mathrm{ppm}\end{array} & \begin{array}{c}2 \sigma \\ \mathrm{ppm}\end{array} & \begin{array}{c}\mathrm{Pb} \\ \mathrm{ppm}\end{array} & \begin{array}{c}2 \sigma \\ \mathrm{ppm}\end{array} & \mathrm{MPb} \\ \text { B2G1-1 } & 578 & 30 & 1824 & 100 & 119262 & 2385 & 3258 & 100 & 207.884 \\ \text { B2G1-2 } & 577 & 32 & 1736 & 100 & 108092 & 2162 & 2952 & 100 & 207.879 \\ \text { B2G1-3 } & 567 & 30 & 1850 & 100 & 116538 & 2331 & 3128 & 100 & 207.880 \\ \text { B2G1-4 } & 587 & 32 & 1900 & 100 & 107293 & 2146 & 2998 & 100 & 207.870 \\ \text { B2G1-5 } & 572 & 32 & 1684 & 100 & 110720 & 2214 & 2989 & 100 & 207.884\end{array}$




\section{Grão B4G2}

$\begin{array}{llllllllllllllllllllllllll}\text { Monaz } & \mathrm{Y}_{2} \mathrm{O}_{3} & \mathrm{Dy}_{2} \mathrm{O}_{3} & \mathrm{P}_{2} \mathrm{O}_{5} & \mathrm{SiO}_{2} & \mathrm{Gd}_{2} \mathrm{O}_{3} & \mathrm{PbO} & \mathrm{FeO} & \mathrm{ThO}_{2} & \mathrm{Sm}_{2} \mathrm{O}_{3} & \mathrm{UO}_{2} & \mathrm{MnO} & \mathrm{CaO} & \mathrm{Nd}_{2} \mathrm{O}_{3} & \mathrm{La}_{2} \mathrm{O}_{3} & \mathrm{Pr}_{2} \mathrm{O}_{3} & \mathrm{Ce}_{2} \mathrm{O}_{3} & \mathrm{Total}\end{array}$ $\begin{array}{llllllllllllllllll}\mathrm{B} 4 \mathrm{G} 2-1 & 2.36 & 0.73 & 29.61 & 0.41 & 1.96 & 0.24 & 0.02 & 4.82 & 2.74 & 1.37 & 0.03 & 1.34 & 11.91 & 11.62 & 3.04 & 28.40 & 100.6\end{array}$ $\begin{array}{lllllllllllllllllll}\text { B4G2-2 } & 2.36 & 0.89 & 29.71 & 0.32 & 2.04 & 0.23 & 0.00 & 4.81 & 2.67 & 1.44 & 0.04 & 1.37 & 11.90 & 11.79 & 2.90 & 28.79 & 101.3\end{array}$ $\begin{array}{llllllllllllllllllll}\text { B4G2-3 } & 2.35 & 0.74 & 29.78 & 0.34 & 1.98 & 0.22 & 0.01 & 5.02 & 2.71 & 1.39 & 0.02 & 1.37 & 12.12 & 11.75 & 3.00 & 28.19 & 100.9\end{array}$

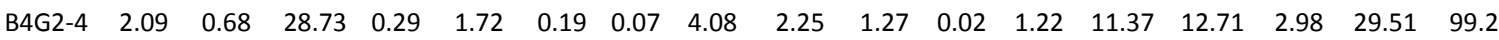

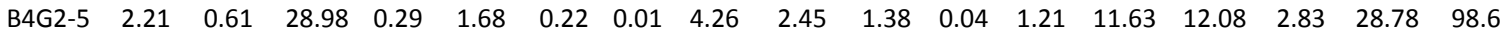

$\begin{array}{lccccccccc}\text { Monaz } & \text { Idade } & 2 \sigma & \mathrm{U} & 2 \sigma & \mathrm{Th} & 2 \sigma & \mathrm{Pb} & 2 \sigma & \mathrm{MPb} \\ & \mathrm{Ma} & \mathrm{Ma} & \mathrm{ppm} & \mathrm{ppm} & \mathrm{ppm} & \mathrm{ppm} & \mathrm{ppm} & \mathrm{ppm} & \\ \text { B4G2-1 } & 596 & 39 & 11842 & 237 & 42376 & 848 & 2182 & 100 & 207.045 \\ \text { B4G2-2 } & 570 & 38 & 12389 & 248 & 42279 & 846 & 2126 & 100 & 207.023 \\ \text { B4G2-3 } & 546 & 38 & 11945 & 239 & 44133 & 883 & 2042 & 100 & 207.062 \\ \text { B4G2-4 } & 539 & 42 & 10949 & 219 & 35855 & 717 & 1736 & 100 & 207.003 \\ \text { B4G2-5 } & 596 & 41 & 11891 & 238 & 37402 & 748 & 2052 & 100 & 206.982\end{array}$

\section{Grão B6G1}

$\begin{array}{llllllllllllllllllllllllll}\text { Monaz } & \mathrm{Y}_{2} \mathrm{O}_{3} & \mathrm{Dy}_{2} \mathrm{O}_{3} & \mathrm{P}_{2} \mathrm{O}_{5} & \mathrm{SiO}_{2} & \mathrm{Gd}_{2} \mathrm{O}_{3} & \mathrm{PbO} & \mathrm{FeO} & \mathrm{ThO}_{2} & \mathrm{Sm}_{2} \mathrm{O}_{3} & \mathrm{UO}_{2} & \mathrm{MnO} & \mathrm{CaO} & \mathrm{Nd}_{2} \mathrm{O}_{3} & \mathrm{La}_{2} \mathrm{O}_{3} & \mathrm{Pr}_{2} \mathrm{O}_{3} & \mathrm{Ce}_{2} \mathrm{O}_{3} & \mathrm{Total}\end{array}$ $\begin{array}{llllllllllllllllllll}\text { B6G1-1 } & 0.84 & 0.35 & 27.70 & 1.61 & 1.33 & 0.31 & 0.02 & 11.63 & 1.98 & 0.47 & 0.03 & 1.64 & 11.20 & 11.53 & 2.85 & 27.67 & 101.1\end{array}$

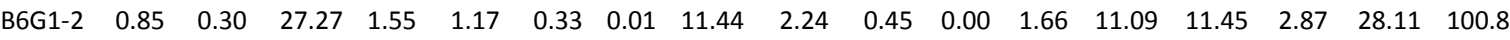

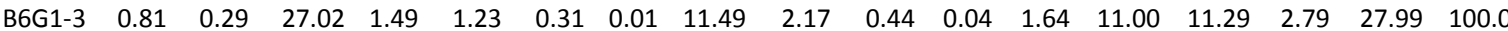

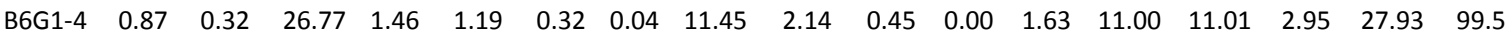

\begin{tabular}{|c|c|c|c|c|c|c|c|c|c|}
\hline Mon & $\begin{array}{c}\text { Idade } \\
\mathrm{Ma}\end{array}$ & $\begin{array}{l}2 \sigma \\
M a\end{array}$ & $\begin{array}{c}\mathrm{U} \\
\mathrm{ppm}\end{array}$ & $\begin{array}{c}2 \sigma \\
\mathrm{ppm}\end{array}$ & $\begin{array}{c}\text { Th } \\
\text { ppm }\end{array}$ & $\begin{array}{c}2 \sigma \\
\mathrm{ppm}\end{array}$ & $\begin{array}{c}\mathrm{Pb} \\
\mathrm{ppm}\end{array}$ & $\begin{array}{c}2 \sigma \\
\mathrm{ppm}\end{array}$ & $M$ \\
\hline $\mathrm{cos}^{2}$ & 560 & 31 & 3475 & 100 & 102231 & 2045 & 2859 & 100 & \\
\hline$G$ & 577 & 32 & 3254 & 100 & 100957 & 2019 & 896 & 00 & 07 \\
\hline $5 G 1$ & 587 & 32 & 3291 & 100 & 100587 & 2012 & 2943 & 100 & 207 \\
\hline 56 & 545 & 31 & 3376 & 100 & 101053 & 2021 & 2748 & 100 & $207.7 \varepsilon$ \\
\hline
\end{tabular}

\section{Grão B7G1}

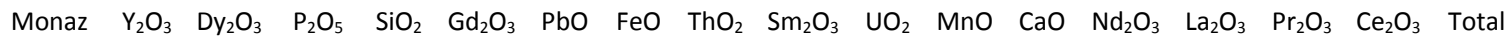

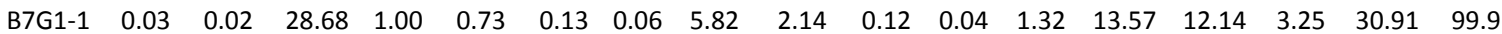
$\begin{array}{llllllllllllllllllll}\text { B7G1-2 } & 0.00 & 0.00 & 28.41 & 0.87 & 0.84 & 0.14 & 0.00 & 5.89 & 2.22 & 0.13 & 0.04 & 1.31 & 13.82 & 12.27 & 3.13 & 31.30 & 100.4\end{array}$ $\begin{array}{llllllllllllllllll}\text { B7G1-3 } & 0.02 & 0.09 & 28.30 & 0.73 & 0.83 & 0.14 & 0.01 & 5.88 & 2.27 & 0.15 & 0.04 & 1.37 & 13.60 & 12.16 & 3.09 & 31.51 & 100.2\end{array}$ $\begin{array}{llllllllllllllllll}\text { B7G1-4 } & 0.20 & 0.05 & 28.31 & 0.61 & 1.11 & 0.14 & 0.01 & 6.19 & 2.56 & 0.18 & 0.03 & 1.86 & 12.97 & 11.88 & 3.08 & 30.93 & 100.1\end{array}$ $\begin{array}{llllllllllllllllll}\text { B7G1-5 } & 0.05 & 0.04 & 27.83 & 0.97 & 0.80 & 0.13 & 0.00 & 5.84 & 2.19 & 0.14 & 0.05 & 1.22 & 13.91 & 12.23 & 3.21 & 32.47 & 101.1\end{array}$

$\begin{array}{lccccccccc}\text { Monaz } & \begin{array}{c}\text { Idade } \\ \text { Ma }\end{array} & \begin{array}{c}\text { Ma } \\ \end{array} & \begin{array}{c}\mathrm{u} p m \\ \text { ppm }\end{array} & \begin{array}{c}2 \sigma \\ \mathrm{ppm}\end{array} & \begin{array}{c}\mathrm{Th} \\ \mathrm{ppm}\end{array} & \begin{array}{c}2 \sigma \\ \mathrm{ppm}\end{array} & \begin{array}{c}\mathrm{Pb} \\ \mathrm{ppm}\end{array} & \begin{array}{c}2 \sigma \\ \text { ppm }\end{array} & \mathrm{MPb} \\ \text { B7G1-1 } & 507 & 54 & 715 & 100 & 51102 & 1022 & 1216 & 100 & 207.891 \\ \text { B7G1-2 } & 519 & 54 & 852 & 100 & 51770 & 1035 & 1272 & 100 & 207.877 \\ \text { B7G1-3 } & 520 & 53 & 958 & 100 & 51682 & 1034 & 1281 & 100 & 207.865 \\ \text { B7G1-4 } & 488 & 50 & 1258 & 100 & 54380 & 1088 & 1281 & 100 & 207.840 \\ \text { B7G1-5 } & 492 & 53 & 890 & 100 & 51357 & 1027 & 1198 & 100 & 207.872\end{array}$

\section{Grão B8G2}

$\begin{array}{lllllllllllllllllllllllllllll}\text { Monaz } & \mathrm{Y}_{2} \mathrm{O}_{3} & \mathrm{Dy}_{2} \mathrm{O}_{3} & \mathrm{P}_{2} \mathrm{O}_{5} & \mathrm{SiO}_{2} & \mathrm{Gd}_{2} \mathrm{O}_{3} & \mathrm{PbO} & \mathrm{FeO} & \mathrm{ThO}_{2} & \mathrm{Sm}_{2} \mathrm{O}_{3} & \mathrm{UO}_{2} & \mathrm{MnO} & \mathrm{CaO} & \mathrm{Nd}_{2} \mathrm{O}_{3} & \mathrm{La}_{2} \mathrm{O}_{3} & \mathrm{Pr}_{2} \mathrm{O}_{3} & \mathrm{Ce}_{2} \mathrm{O}_{3} & \mathrm{Total}\end{array}$ $\begin{array}{lllllllllllllllllll}\mathrm{B} 8 \mathrm{G} 2-1 & 0.11 & 0.00 & 30.15 & 0.95 & 0.49 & 0.16 & 0.02 & 6.12 & 1.56 & 0.28 & 0.00 & 1.01 & 11.38 & 14.95 & 3.08 & 30.74 & 100.9\end{array}$ $\begin{array}{llllllllllllllllll}\text { B8G2-2 } & 0.14 & 0.03 & 29.00 & 0.91 & 0.56 & 0.19 & 0.03 & 6.93 & 1.64 & 0.32 & 0.01 & 1.07 & 11.44 & 14.70 & 3.08 & 30.71 & 100.8\end{array}$ $\begin{array}{lllllllllllllllllll}\text { B8G2-3 } & 0.07 & 0.03 & 29.18 & 0.86 & 0.51 & 0.19 & 0.01 & 7.14 & 1.52 & 0.31 & 0.01 & 1.07 & 11.04 & 14.83 & 3.06 & 31.04 & 100.9\end{array}$ $\begin{array}{llllllllllllllllllll}\text { B8G2-4 } & 0.10 & 0.10 & 29.03 & 0.93 & 0.52 & 0.17 & 0.01 & 6.22 & 1.68 & 0.31 & 0.02 & 1.00 & 11.54 & 14.64 & 3.13 & 30.65 & 100.0\end{array}$ $\begin{array}{llllllllllllllllll}\text { B8G2-5 } & 0.03 & 0.03 & 29.40 & 0.73 & 0.61 & 0.14 & 0.00 & 5.19 & 1.87 & 0.24 & 0.02 & 0.99 & 12.45 & 14.37 & 3.30 & 32.47 & 101.9\end{array}$ $\begin{array}{llllllllllllllllll}\text { B8G2-6 } & 0.13 & 0.00 & 28.62 & 1.32 & 0.52 & 0.21 & 0.03 & 7.87 & 1.51 & 0.27 & 0.01 & 0.93 & 10.35 & 15.01 & 2.58 & 31.71 & 101.1\end{array}$

$\begin{array}{lccccccccc}\text { Monaz } & \begin{array}{c}\text { Idade } \\ \text { Ma }\end{array} & \begin{array}{c}\text { Ma } \\ \text { Ma }\end{array} & \begin{array}{c}\mathrm{ppm} \\ \mathrm{ppm}\end{array} & \begin{array}{c}2 \sigma \\ \mathrm{ppm}\end{array} & \begin{array}{c}\text { Th } \\ \mathrm{ppm}\end{array} & \begin{array}{c}2 \sigma \\ \mathrm{ppm}\end{array} & \begin{array}{c}\mathrm{Pb} \\ \mathrm{ppm}\end{array} & \begin{array}{c}2 \sigma \\ \mathrm{ppm}\end{array} & \mathrm{MPb} \\ \text { B8G2-1 } & 547 & 49 & 2126 & 100 & 53818 & 1076 & 1495 & 100 & 207.754 \\ \text { B8G2-2 } & 561 & 45 & 2416 & 100 & 60874 & 1217 & 1736 & 100 & 207.752 \\ \text { B8G2-3 } & 552 & 44 & 2307 & 100 & 62711 & 1254 & 1745 & 100 & 207.767 \\ \text { B8G2-4 } & 546 & 48 & 2358 & 100 & 54653 & 1093 & 1532 & 100 & 207.735 \\ \text { B8G2-5 } & 576 & 57 & 1808 & 100 & 45619 & 912 & 1337 & 100 & 207.753 \\ \text { B8G2-6 } & 563 & 42 & 1922 & 100 & 69179 & 1384 & 1912 & 100 & 207.814\end{array}$



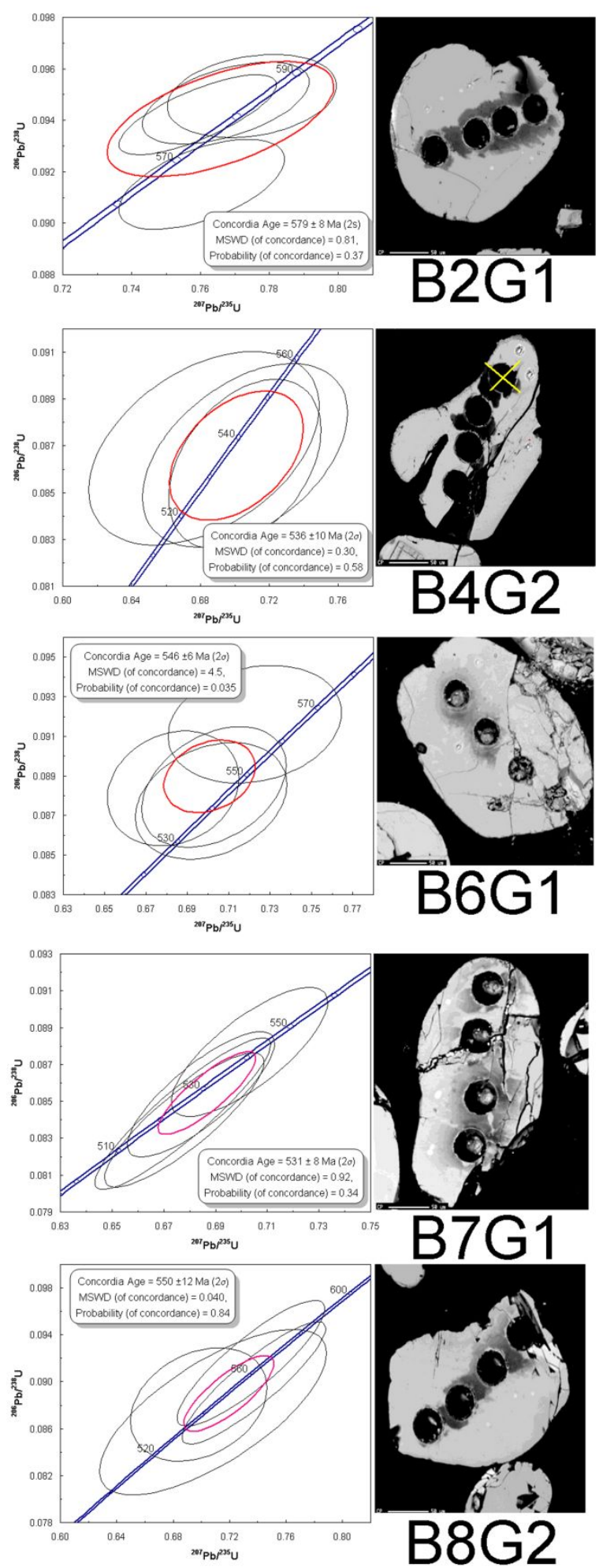

Figura 1 - Idades concordantes U-Pb para os 5 grãos de monazita investigados e suas respectivas imagens de elétrons retroespalhados. Notar os buracos deixados pela ablação a laser.

\section{DISCUSSÕES E CONCLUSÕES}

A tabela 4 a seguir apresenta o resumo comparativo das idades isotópicas e não-isotópicas dos grãos de monazita investigados.
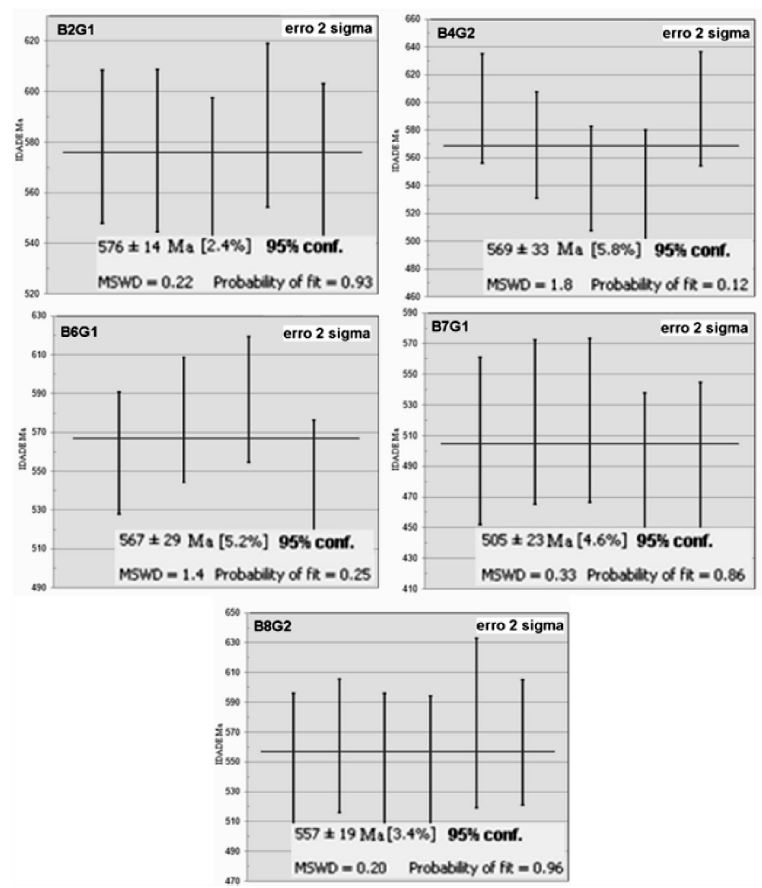

Figura 2: Média das idades químicas U-Th-Pb (não-isotópicas) dos 5 grãos de monazita.

Tabela 4 - Quadro comparativo das idades isotópicas $U$-Pb e idades químicas U-Th-Pb dos grãos de monazita de Buena-RJ. Erros de $2 \sigma$.

\begin{tabular}{|c|c|c|}
\hline Monazita & Idade isotópica U-Pb (Ma) & Idade química U-Th-Pb (Ma) \\
\hline B2G1 & $579+/-8$ & $576+/-14$ \\
\hline B4G2 & $536+/-10$ & $569+/-33$ \\
\hline B6G1 & $546+/-6$ & $567+/-29$ \\
\hline B7G1 & $531+/-8$ & $505+/-23$ \\
\hline B8G2 & $550+/-12$ & $557+/-19$ \\
\hline
\end{tabular}

Considerando os erros das idades, todas as idades médias não-isotópicas dos grãos de monazita estudados que foram obtidas pelo método de datação química U-Th-Pb em microssonda eletrônica encaixam-se nas idades isotópicas $\mathrm{U}-\mathrm{Pb}$ correspondentes. Um maior número de análises por cristal de monazita poderia, eventualmente, diminuir estatisticamente as pequenas diferenças de idade verificadas. A semelhança dos resultados confirma a robustez do método não-isotópico de datação de monazita, já consagrado na literatura geológica, e coloca o LMA-UFMG à disposição da comunidade geocientífica brasileira como uma alternativa à obtenção de dados geocronológicos confiáveis para este mineral, sob a condição de que seja monazita portadora de Th e eventualmente $U$, bem como do $\mathrm{Pb}$ radiogênico deles derivado.

\section{AGRADECIMENTOS}

À INB pelo fornecimento do concentrado de monazita e ao Prof. B. Buhn (UnB) pelo apoio na obtenção das idades isotópicas U-Pb. Em especial, ao Coordenador do LMA-UFMG, Prof. Abá Israel Cohen Persiano, pelo irrestrito apoio e interesse no desenvolvimento do método em questão. 


\section{REFERÊNCIAS}

Albarède F., Telouk P., Blichert-Toft J., Boyet M., Agranier A. Nelson B. 2004. Precise and accurate isotopic measurements using multiple-collector ICPMS. Geochimica et Cosmochimica Acta, 68:2725-2744.

Bühn B., Pimentel M.M., Matteini M., Dantas E.L. 2009. High spatial resolution analysis of $\mathrm{Pb}$ and $U$ isotopes for geochronology by laser ablation multi-collector inductively coupled plasma mass spectrometry (LA-MC-ICP-MS). Anais da Academia Brasileira de Ciências, 81:99-114.

Dahl P.S., Hamilton M.A., Jercinovic M.J., Terry M.P., Williams M.L., Frei, R., 2005. Comparative isotopic and chemical geochronometry of monazite, with implications for $\mathrm{U}-\mathrm{Th}-\mathrm{Pb}$ dating by electron microprobe: An example from metamorphic rocks of the eastern Wyoming Craton (U.S.A.). American Mineralogist, 90: 619-638.

Foster G., Parrish R.R., Horstwood, M.S., Chenery, S., Pyle, J., and Gibson, H.D., 2004. The generation of prograde P-T-t points and paths; a textural, compositional, and chronological study of metamorphic monazite. Earth Planetary Science Letters, 228: 125-142.

Jackson S.E., Pearson N.J., Griffin W.L., Belousova E.A. 2004. The application of laser ablation inductively coupled plasma mass spectrometry to in situ $\mathrm{U}-\mathrm{Pb}$ zircon geochronology. Chemical Geology, 211: 47-69.

Ludwig K. R. 2003. Isoplot/Ex 3.00: A geochronological toolkit for Microsoft Excel. Berkeley Geochronology Center, Special Publication, n.4, 70 p.

Montel J., Foret S., Veschambre M., Nicollet C., Provost A., 1996. Electron microprobe dating of monazite. Chemical Geology. 131: 37- 53 .

Overstreet W.C., 1967. The geological occurrence of monazite. U.S. Geological Survey Professional Papers. 530, 327.
Parrish R.R. 1990. U-Pb dating of monazite and its applications to geological problems. Canadian Journal of Earth Science, 27: 1431-1450

Pommier A.,Cocherie A., Legendre O. 2004. EPMA Dating User' Manual, V.1.01: Age Calculation from Electron Probe Microanalyser Measurements of U-Th-Pb. BRGM, Orleans.

Pyle J.M., Spear F.S., Wark D.A., Daniel C.G., Storm L.C. 2005. Contributions to precision and accuracy of chemical ages of monazite. American Mineralogist, 90: 547-577.

Scherrer N.C., Eng M., Gnos E., Jakob V., Liechti A. 2000. Monazite analysis; from sample preparation to microprobe age dating and REE quantification. Schweizer Mineralogische und Petrographische Mitteilungen, 80: 93-105.

Stacey J.S. e Kramers J.D. 1975. Approximation of terrestrial lead isotope evolution by a two-stage model. Earth and Planetary Science Letters, 26: 207-221.

Suzuki K. e Adachi M., 1991. Precambrian provenance and Silurian metamorphism of the Tsubonosawa paragneiss in the South Kitakami terrane, Northeast Japan, revealed by the chemical Th-U-total $\mathrm{Pb}$ isochron ages of monazite, zircon, and xenotime. Geochemical Journal. 25: 357- 376.

Toya T., Kato A., Jotaki R. 1984. Quantitative Analysis with Electron Probe Microanalyzer. Jeol Training Center. Japan. $113 p$.

Vlach S.R.F. 2010.Th-U-Pb T $_{\text {T }}$ Dating by Electron Probe Microanalysis, Part I. Monazite: Analytical Procedures and Data Treatment. Geologia USP-Série científica,10(1): 61-85.

Williams M.L., Jercinovic M.J., Terry M.P. 1999. Age mapping and dating of monazite on the electron microprobe: Deconvoluting multistage tectonic histories. Geology 27: 1023-1026.

Williams M.L., Jercinovic M.J., Hetherington CJ. 2007. Microprobe monazite geochronology: understanding geologic processes by integrating composition and chronology. Ann Review Earth Planetary Sciences. 35: 137-175. 